International Journal of Physical Research, 9(2)(2021) $98-104$
International Journal of Physical Research
SPC
Website: www.sciencepubco.com/index.php/IJPR
Research paper

\title{
Periodic wave analysis to the time-fractional phi-four and (2+1) dimensional CBS equations
}

\author{
Samsun Nahar Ananna ${ }^{1}$, Abdulla - Al - Mamun 1, 2, 3 *, Tianqing An ${ }^{3}$ \\ ${ }^{1}$ Department of Mathematics, College of Science, Hohai University, Nanjing-210098, PR China \\ ${ }^{2}$ Department of Information and Communication Technology, Rabindra Maitree University, Kushtia-7000, Bangladesh \\ ${ }^{3}$ Department of Hydrology and Water Resources, College of Hydrology and Water Resources, Hohai \\ University, Nanjing-210098, PR China \\ *Corresponding author E-mail: abdullamamun21@gmail.com
}

\begin{abstract}
In this paper, we investigate some innovative and exact travelling wave solutions to the time-fractional phi-four equation and the (2+1) dimensional Calogero-Bogoyavlanskil schilf (CBS) equation using the $\left(G^{\prime} / G^{2}\right)$-expansion method. Periodic solutions are displayed in hyperbolic, trigonometric, and rational function solutions. Subsequently, we construct some new solutions connecting the free parameters of the phi-four equation and the CBS equation, which are characterized into three complete forms: rational function, trigonometric function, and hyperbolic functions. Graphical representations of some attained solutions are also presented in this article. Hence, this study shows the efficiency and the easiness of the $\left(G^{\prime} / G^{2}\right)$-expansion technique with the assistance of emblematically computational software MATLAB and Mathematica.
\end{abstract}

Keywords: The $\left(G^{\prime} / G^{2}\right)$-Expansion Technique; Travelling Wave Solution; Phi-Four Equation; Periodic Wave; Calogero-Bogoyavlanskil Schilf Equation.

\section{Introduction}

In engineering, fluid dynamics, plasma physics, applied mathematics, and mathematical physics, nonlinear fractional partial differential equations (FPDEs) model significant phenomena and applications. To obtain some new analytical solutions, many authors absorbed enhanced new approaches or adjustments to standing systems. To find traveling wave solutions, efforts have increased in the literature of practical mathematics. It is broadly used to perceive the complex physical amplification of mathematical physics, nuclear physics, plasma physics, solid-state physics, statistical physics, astrophysics, mechanical engineering, fractional dynamics, biomechanics, strong state material science, neural material science, fluid mechanics, geo-optical filaments, stochastic dynamics, nonlinear optics, etcetera, etc. [1]. Thus, many influential and effective approaches have been established to find analytical solutions for traveling waves, such as the modified extended tanh-function technique, [2]-[4], the advanced $\exp (-\phi(\psi))$-expansion technique, [5],[6], the $\left(G^{\prime} / G, 1 / G\right)$-expansion technique, [7],[8], method of characteristics, [9], the variational iteration process, [10]-[12], the improved simple equation system, [13], the novel exponential rational function technique, [14], the multiple Exp-function system, [15],[16], the improved $\tan (\phi(\xi) / 2)$ and $\tanh (\phi(\xi) / 2)$-expansion approaches, [17],[18], the Darboux transform process, [19], the exponential rational function technique, [20], extended simple equation method, [21], the updated simple equation method, [22], the Hirota bilinear method, [23], the first integral method, [24], the expanded trial equation method, [25], symbolic computations, [26],[27], a transformed rational function technique, [28], the ansatz structure, [29], the sine-cosine system, [30], the new extended direct algebraic scheme, [31], the $\left(G^{\prime} / G^{2}\right)$-expansion technique, [32], the $\left(\mathrm{G}^{\prime} / \mathrm{G}\right)$-expansion technique, [33], etc.

The goal of this study is to develop precise travelling waves analysis using the $\left(G^{\prime} / G^{2}\right)$-expansion technique to better understand the physical meaning of a diversity of the phi-four and CBS equations. The unique solutions eliminated by the $\left(\mathrm{G}^{\prime} / \mathrm{G}^{2}\right)$-expansion approach is articulated by the arrangement of the sinh, cosh, sin, and cos functions. Specify periodic waves solutions when creating joint solutions. The remainder of the paper is prepared in the following manner. The $\left(G^{\prime} / G^{2}\right)$-expansion approach is briefly designated in Section 2 . In Section 3, the mathematical formulation of the phi-four and CBS equations is provided, as well as its application using the $\left(\mathrm{G}^{\prime} / \mathrm{G}^{2}\right)$ expansion technique. Section 4 is where this paper's findings are drawn.

\section{Explanation of the $\left(G^{\prime} / G^{2}\right)$-expansion technique}

In this part, the $\left(G^{\prime} / G^{2}\right)$-expansion technique is discoursed and assessed using the recommended methodology.

Suppose a nonlinear FDE presumed by

$\mathrm{F}\left(\mathrm{u}, \mathrm{D}_{\mathrm{t}}^{\gamma} \mathrm{u}, \mathrm{D}_{\mathrm{x}}^{\gamma} \mathrm{u}, \mathrm{D}_{\mathrm{y}}^{\gamma} \mathrm{u}, \mathrm{D}_{\mathrm{t}}^{\gamma} \mathrm{D}_{\mathrm{t}}^{\gamma} \mathrm{u}, \mathrm{D}_{\mathrm{t}}^{\gamma} \mathrm{D}_{\mathrm{x}}^{\gamma} \mathrm{u}, \mathrm{D}_{\mathrm{x}}^{\gamma} \mathrm{D}_{\mathrm{x}}^{\gamma} \mathrm{u}\right)=0,0<\gamma<1$.

Copyright () Samsun Nahar Ananna et al. This is an open access article distributed under the Creative Commons Attribution License, which permits unrestricted use, distribution, and reproduction in any medium, provided the original work is properly cited. 
In the previous FDE, $\mathrm{u}(\mathrm{x}, \mathrm{y}, \mathrm{t})$ is an enigmatic purpose, and $\mathrm{F}$ is a polynomial of $\mathrm{u}$ and partial fractional derivatives.

By disbursing the complex fractional transformation, we acquire.

$$
\begin{gathered}
\mathrm{u}(\mathrm{x}, \mathrm{t})=\mathrm{U}(\psi), \\
\psi=\mathrm{p} \frac{\mathrm{x}^{\gamma}}{\gamma}+\mathrm{q} \frac{\mathrm{y}^{\gamma}}{\gamma}+\mathrm{r} \frac{\mathrm{z}^{\gamma}}{\gamma}-\mathrm{s} \frac{\mathrm{t}^{\gamma}}{\gamma},
\end{gathered}
$$

Where p, q, r, and s are nonzero constants. Equation (2.2) can be distorted into an ODE of the form:

$\mathrm{Q}\left(\mathrm{U}, \mathrm{U}^{\prime}, \mathrm{U}^{\prime \prime}, \mathrm{U}^{\prime \prime \prime}, \ldots \ldots\right)=0$,

The formula explanation of ODE can be written as:

$$
\begin{aligned}
& U(\psi)=A_{0}+\sum_{k=1}^{N}\left[A_{k}\left(\frac{G^{\prime}}{G^{2}}\right)^{k}+B_{k}\left(\frac{G^{\prime}}{G^{2}}\right)^{-k}\right], \\
& \left(\frac{G^{\prime}}{G^{2}}\right)^{\prime}=\mu+\lambda\left(\frac{G^{\prime}}{G^{2}}\right)^{2},
\end{aligned}
$$

Where, $\lambda \neq 0$, and $A_{0}, A_{k}, B_{k}(k=1,2,3, \ldots \ldots, N)$ are constants to be determined.

Based on the general solutions to equation (2.5), the ratio $\left(\frac{G^{\prime}}{G^{2}}\right)$ can be separated into three cases as follows:

Case-I. Hyperbolic function solution, when $(\mu \lambda<0)$

$\frac{\mathrm{G}^{\prime}}{\mathrm{G}^{2}}=-\frac{\sqrt{|\mu \lambda|}}{\lambda}\left[\frac{\mathrm{a} \sinh (2 \sqrt{\mu \lambda} \psi)+\mathrm{a} \cosh (2 \sqrt{\mu \lambda} \psi)+\mathrm{b}}{\mathrm{a} \sinh (2 \sqrt{\mu \lambda} \psi)+\mathrm{a} \cosh (2 \sqrt{\mu \lambda} \psi)-\mathrm{b}}\right]$

Case-II. Rational function solution, when $(\mu \lambda=0)$

$\frac{\mathrm{G}^{\prime}}{\mathrm{G}^{2}}=-\frac{\mathrm{a}}{\lambda(\mathrm{a} \psi+\mathrm{b})}, \mu=0, \lambda \neq 0$,

Case-III. Trigonometric function solution, when $(\mu \lambda>0)$

$\frac{G^{\prime}}{G^{2}}=\sqrt{\frac{\mu}{\lambda}}\left[\frac{\mathrm{a} \cos (\sqrt{\mu \lambda} \psi)+b \sin (\sqrt{\mu \lambda} \psi)}{b \cos (\sqrt{\mu \lambda} \psi)-a \sin (\sqrt{\mu \lambda} \psi)}\right]$,

Where $\mathrm{a}, \mathrm{b} \neq 0$ for all three cases.

\section{Applications of the $\left(G^{\prime} / G^{2}\right)$-expansion technique}

\subsection{The nonlinear time-fractional Phi-four equation}

The Phi-four equation is a specific form of the Klein-Gordon equation.

$D_{t}^{2 \theta}-u_{x x}+\beta^{2} u+\gamma u^{3}=0, \gamma>0,0<\theta \leq 1$

Where $\beta$ and $\gamma$ are real numbers.

Employing the following travelling wave transformation

$\mathrm{u}(\mathrm{x}, \mathrm{t})=\mathrm{U}(\psi)$, where $\psi=\mathrm{qx}-\mathrm{p} \frac{\mathrm{t}^{\theta}}{\theta}$

On Eq. (3.1), we get

$\left(p^{2}-q^{2}\right) U^{\prime \prime}+\beta^{2} U+\gamma U^{3}=0$,

With the asset of homogeneous balancing of the highest order derivative term $\mathrm{U}^{\prime \prime}$ and nonlinear term $\mathrm{U}^{3}$ in Eq. 3.2 , we find that $\mathrm{N}=1$. Thus, our recommended technique allows us to use the auxiliary equation of the form:

$\mathrm{U}(\psi)=\mathrm{A}_{0}+\mathrm{A}_{1} \alpha+\mathrm{B}_{1} \alpha^{-1}$

Now putting the value of $U$ and $U^{\prime \prime}$ in Eq. (3.2), we get,

$\beta^{2} A_{0}+\gamma A_{0}^{3}+G \beta^{2} A_{1}+2 G^{3} p^{2} \lambda^{2} A_{1}-2 G^{3} q^{2} \lambda^{2} A_{1}+2 G p^{2} \lambda \mu A_{1}-2 G q^{2} \lambda \mu A_{1}+3 G \gamma A_{0}^{2} A_{1}+3 G^{2} \gamma A_{0} A_{1}^{2}+G^{3} \gamma A_{1}^{3}+\frac{\beta^{2} B_{1}}{G}+\frac{2 p^{2} \lambda \mu B_{1}}{G}-$ $\frac{2 q^{2} \lambda \mu B_{1}}{G}+\frac{2 p^{2} \mu^{2} B_{1}}{G^{3}}-\frac{2 q^{2} \mu^{2} B_{1}}{G^{3}}+\frac{3 \gamma A_{0}^{2} B_{1}}{G}+6 \gamma A_{0} A_{1} B_{1}+3 G \gamma A_{1}^{2} B_{1}+\frac{3 \gamma A_{0} B_{1}^{2}}{G^{2}}+\frac{3 \gamma A_{1} B_{1}^{2}}{G}+\frac{\gamma B_{1}^{3}}{G^{3}}=0$

Compeering the coefficients of like power of $\alpha$ from both sides of the above equation, we get the following SAE: 


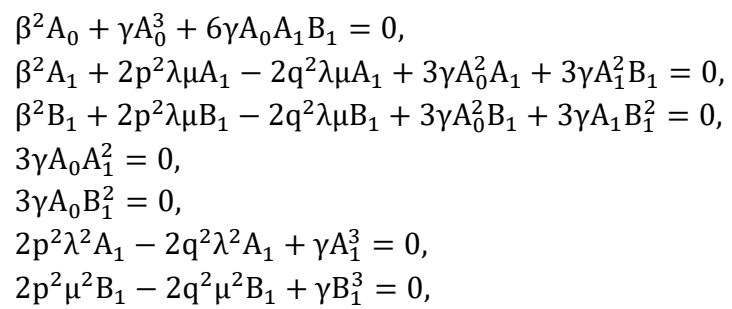

Solving the SAE Eq. (3.4) for $p, A_{0}, A_{1}, B_{1}$ we get several solutions sets as follows:

$$
\begin{aligned}
& q= \pm \frac{\sqrt{\beta^{2}+2 p^{2} \lambda \mu}}{\sqrt{2} \sqrt{\lambda} \sqrt{\mu}}, A_{0}=0, A_{1}= \pm \frac{\beta \sqrt{\lambda}}{\sqrt{\gamma} \sqrt{\mu}}, B_{1}=0 . \\
& q= \pm \frac{\sqrt{\beta^{2}+2 p^{2} \lambda \mu}}{\sqrt{2} \sqrt{\lambda} \sqrt{\mu}}, A_{0}=0, A_{1}=0, B_{1}= \pm \frac{\beta \sqrt{\mu}}{\sqrt{\gamma} \sqrt{\lambda}} . \\
& q= \pm \frac{\sqrt{-\beta^{2}+4 p^{2} \lambda \mu}}{2 \sqrt{\lambda} \sqrt{\mu}}, A_{0}=0, A_{1}=-\frac{i \beta \sqrt{\lambda}}{\sqrt{2} \sqrt{\gamma} \sqrt{\mu}}, B_{1}=-\frac{i \beta \sqrt{\mu}}{\sqrt{2} \sqrt{\gamma} \sqrt{\lambda}} \\
& q= \pm \frac{\sqrt{-\beta^{2}+4 p^{2} \lambda \mu}}{2 \sqrt{\lambda} \sqrt{\mu}}, A_{0}=0, A_{1}=\frac{i \beta \sqrt{\lambda}}{\sqrt{2} \sqrt{\gamma} \sqrt{\mu}}, B_{1}=\frac{i \beta \sqrt{\mu}}{\sqrt{2} \sqrt{\gamma} \sqrt{\lambda}} . \\
& q= \pm \frac{\sqrt{\beta^{2}+8 p^{2} \lambda \mu}}{2 \sqrt{2} \sqrt{\lambda} \sqrt{\mu}}, A_{0}=0, A_{1}=-\frac{\beta \sqrt{\lambda}}{2 \sqrt{\gamma} \sqrt{\mu}}, B_{1}=\frac{\beta \sqrt{\mu}}{2 \sqrt{\gamma} \sqrt{\lambda}} . \\
& q= \pm \frac{\sqrt{\beta^{2}+8 p^{2} \lambda \mu}}{2 \sqrt{2} \sqrt{\lambda} \sqrt{\mu}}, A_{0}=0, A_{1}=\frac{\beta \sqrt{\lambda}}{2 \sqrt{\gamma} \sqrt{\mu}}, B_{1}=-\frac{\beta \sqrt{\mu}}{2 \sqrt{\gamma} \sqrt{\lambda}} .
\end{aligned}
$$

Expending these solution sets, we build the solutions to Eq. (3.1) as follows:

When $\mu \lambda<0$, we obtain the following hyperbolic function solutions:

$$
\begin{aligned}
& U_{1,2}(x, t)= \pm \beta \sqrt{\frac{|\lambda \mu|}{\gamma \lambda \mu}}\left[\frac{b+a \operatorname{Cosh}[2 \sqrt{\lambda \mu} \psi]+a \operatorname{Sinh}[2 \sqrt{\lambda \mu} \psi]}{-b+a \operatorname{Cosh}[2 \sqrt{\lambda \mu} \psi]+a \operatorname{Sinh}[2 \sqrt{\lambda \mu} \psi]}\right] \psi=q x-p \frac{t^{\theta}}{\theta} ; q= \pm \frac{\sqrt{\beta^{2}+2 p^{2} \lambda \mu}}{\sqrt{2} \sqrt{\lambda} \sqrt{\mu}} \\
& U_{3,4}(x, t)= \pm \beta \sqrt{\frac{\mu \lambda}{\gamma|\lambda \mu|}\left[\frac{-b+a \operatorname{Cosh}[2 \sqrt{\lambda \mu} \psi]+a \operatorname{Sinh}[2 \sqrt{\lambda \mu} \psi]}{b+a \operatorname{Cosh}[2 \sqrt{\lambda \mu} \psi]+a \operatorname{Sinh}[2 \sqrt{\lambda \mu} \psi]}\right] \psi=q x-p \frac{t^{\theta}}{\theta} ; q= \pm \frac{\sqrt{\beta^{2}+2 p^{2} \lambda \mu}}{\sqrt{2} \sqrt{\lambda} \sqrt{\mu}}} \\
& U_{5,6}(x, t)= \pm \frac{\mathrm{i} \beta\left(\mu+\sqrt{|\lambda \mu|}\left[\frac{b+a \operatorname{Cosh}[2 \sqrt{\lambda \mu} \psi]+a \operatorname{Sinh}[2 \sqrt{\lambda \mu} \psi]}{-b+a \operatorname{Cosh}[2 \sqrt{\lambda \mu} \psi]+a \operatorname{Sinh}[2 \sqrt{\lambda \mu} \psi]}\right]\right)}{\sqrt{\frac{2 \gamma \mu|\lambda \mu|}{\lambda}}\left[\frac{b+a \operatorname{Cosh}[2 \sqrt{\lambda \mu} \psi]+a \operatorname{Sinh}[2 \sqrt{\lambda \mu} \psi]}{-b+a \operatorname{Cosh}[2 \sqrt{\lambda \mu} \psi]+a \operatorname{Sinh}[2 \sqrt{\lambda \mu} \psi]}\right]} ; \psi=q x-p \frac{t^{\theta}}{\theta} ; q= \pm \frac{\sqrt{-\beta^{2}+4 p^{2} \lambda \mu}}{2 \sqrt{\lambda} \sqrt{\mu}} \\
& U_{7,8}(x, t)= \pm \frac{\beta\left(\mu-\sqrt{|\lambda \mu|}\left[\frac{b+a \operatorname{Cosh}[2 \sqrt{\lambda \mu} \psi]+a \operatorname{Sinh}[2 \sqrt{\lambda \mu} \psi]}{-b+a \operatorname{Cosh}[2 \sqrt{\lambda \mu} \psi]+a \operatorname{Sinh}[2 \sqrt{\lambda \mu} \psi]}\right]\right)}{\sqrt{\frac{2 \gamma \mu|\lambda \mu|}{\lambda}}\left[\frac{b+a \operatorname{Cosh}[2 \sqrt{\lambda \mu} \psi]+a \operatorname{Sinh}[2 \sqrt{\lambda \mu} \psi]}{-b+a \operatorname{Cosh}[2 \sqrt{\lambda \mu} \psi]+a \operatorname{Sinh}[2 \sqrt{\lambda \mu} \psi]}\right]} ; \psi=q x-p \frac{t^{\theta}}{\theta} ; q= \pm \frac{\sqrt{\beta^{2}+8 p^{2} \lambda \mu}}{2 \sqrt{2} \sqrt{\lambda} \sqrt{\mu}}
\end{aligned}
$$

When $\mu \lambda>0$, we obtain the following trigonometric function solution

$$
\begin{aligned}
& U_{9,10}(x, t)= \pm \frac{\beta}{\sqrt{\gamma}}\left[\frac{a \operatorname{Cos}[\sqrt{\lambda \mu} \psi]+b \operatorname{Sin}[\sqrt{\lambda \mu} \psi]}{b \operatorname{Cos}[\sqrt{\lambda \mu} \psi]-a \operatorname{Sin}[\sqrt{\lambda \mu} \psi]}\right] ; \psi q x-p \frac{t^{\theta}}{\theta} ; q= \pm \frac{\sqrt{\beta^{2}+2 p^{2} \lambda \mu}}{\sqrt{2} \sqrt{\lambda} \sqrt{\mu}} \\
& U_{11,12}(x, t)= \pm \frac{\beta}{\sqrt{\gamma}}\left[\frac{b \operatorname{Cos}[\sqrt{\lambda \mu} \psi]-a \operatorname{Sin}[\sqrt{\lambda \mu} \psi]}{a \operatorname{Cos}[\sqrt{\lambda \mu} \psi]+b \operatorname{Sin}[\sqrt{\lambda \mu} \psi]}\right] ; \psi=q x-p \frac{t^{\theta}}{\theta} ; q= \pm \frac{\sqrt{\beta^{2}+2 p^{2} \lambda \mu}}{\sqrt{2} \sqrt{\lambda} \sqrt{\mu}} \\
& U_{13,14}(x, t)= \pm \frac{\mathrm{i} \beta\left(\mu+\sqrt{\lambda \mu}\left[\frac{a \operatorname{Cos}[\sqrt{\lambda \mu} \psi]+b \operatorname{Sin}[\sqrt{\lambda \mu} \psi]}{b \operatorname{Cos}[\sqrt{\lambda \mu} \psi]-a \operatorname{Sin}[\sqrt{\lambda \mu} \psi]}\right]\right)}{\mu \sqrt{2 \gamma}\left[\frac{a \operatorname{Cos}[\sqrt{\lambda \mu} \psi]+b \operatorname{Sin}[\sqrt{\lambda \mu} \psi]}{b \operatorname{Cos}[\sqrt{\lambda \mu} \psi]-a \operatorname{Sin}[\sqrt{\lambda \mu} \psi]}\right]} ; \psi=q x-p \frac{t^{\theta}}{\theta} ; q= \pm \frac{\sqrt{-\beta^{2}+4 p^{2} \lambda \mu}}{2 \sqrt{\lambda} \sqrt{\mu}}
\end{aligned}
$$


$U_{15,16}(x, t)= \pm \frac{\beta\left(\mu-\sqrt{\lambda \mu}\left[\frac{a \operatorname{Cos}[\sqrt{\lambda \mu} \psi]+b \operatorname{Sin}[\sqrt{\lambda \mu} \psi]}{b \operatorname{Cos}[\sqrt{\lambda \mu} \psi]-a \operatorname{Sin}[\sqrt{\lambda \mu} \psi]}\right]^{2}\right)}{2 \mu \sqrt{\gamma}\left[\frac{a \operatorname{Cos}[\sqrt{\lambda \mu} \psi]+b \operatorname{Sin}[\sqrt{\lambda \mu} \psi]}{b \operatorname{Cos}[\sqrt{\lambda \mu} \psi]-a \operatorname{Sin}[\sqrt{\lambda \mu} \psi]}\right]} ; \psi=q x-p \frac{t^{\theta}}{\theta} ; q= \pm \frac{\sqrt{\beta^{2}+8 p^{2} \lambda \mu}}{2 \sqrt{2} \sqrt{\lambda} \sqrt{\mu}}$

\subsection{The nonlinear time-fractional CBS equation}

Consider the subsequent generalized (2+1)-dimensional CBS circumstances:

$u_{t}+\phi(u) u_{y}=0, \phi(u)=\partial_{x}^{2}+a u+b u_{x} \partial_{x}^{-1}$,

Or homogeneously,

$u_{t}+u_{x x y}+a u u_{y}+b v_{x} \partial_{x}^{-1} v_{y}=0$

Where $\partial_{x}^{-1}=\int f d x$ and $\mathrm{a}, \mathrm{b}$ are constraints. Eq. (3.6) can be characterized in the probable time-fractional form of CBS equation. ${ }^{2,31}$

$u_{x} D_{t}^{\theta} \theta_{u}+4 u_{x} u_{x y}+2 u_{x x} u_{y}+u_{x x x y}=0, t>0, x, y \in \mathbb{R}$

Where $0<\theta \leq 1$.

Employing the following travelling wave transformation

$u(x, y, t)=U(\psi)$ and $\psi=x+y-p \frac{t^{\theta}}{\theta}$

On Eq. (3.7), we get

$p U^{\prime}+\left(\frac{\beta+\gamma}{2}\right)\left(U^{\prime}\right)^{2}+U^{\prime \prime \prime}=0$

With the asset of homogeneous balancing of the highest order derivative term $U^{\prime \prime \prime}$ and the nonlinear term $\left(U^{\prime}\right)^{2}$ in Eq. $(3.9)$, we find that $N=1$. Thus, our recommended technique allows us to use the auxiliary equation of the form:

$U(\psi)=A_{0}+A_{1} \alpha+B_{1} \alpha^{-1}$

From Eq. (3.9) and (3.10) we get,

$-G^{2} p \lambda A_{1}+6 G^{4} \lambda^{3} A_{1}-p \mu A_{1}+8 G^{2} \lambda^{2} \mu A_{1}+2 \lambda \mu^{2} A_{1}+\frac{1}{2} G^{4} \beta \lambda^{2} A_{1}^{2}+\frac{1}{2} G^{4} \gamma \lambda^{2} A_{1}^{2}+G^{2} \beta \lambda \mu A_{1}^{2}+G^{2} \gamma \lambda \mu A_{1}^{2}+\frac{1}{2} \beta \mu^{2} A_{1}^{2}+\frac{1}{2} \gamma \mu^{2} A_{1}^{2}+$ $p \lambda B_{1}+\frac{p \mu B_{1}}{G^{2}}-2 \lambda^{2} \mu B_{1}-\frac{8 \lambda \mu^{2} B_{1}}{G^{2}}-\frac{6 \mu^{3} B_{1}}{G^{4}}-G^{2} \beta \lambda^{2} A_{1} B_{1}-G^{2} \gamma \lambda^{2} A_{1} B_{1}-2 \beta \lambda \mu A_{1} B_{1}-2 \gamma \lambda \mu A_{1} B_{1}-\frac{\beta \mu^{2} A_{1} B_{1}}{G^{2}}-\frac{\gamma \mu^{2} A_{1} B_{1}}{G^{2}}+\frac{1}{2} \beta \lambda^{2} B_{1}^{2}+$ $\frac{1}{2} \gamma \lambda^{2} B_{1}^{2}+\frac{\beta \lambda \mu B_{1}^{2}}{G^{2}}+\frac{\gamma \lambda \mu B_{1}^{2}}{G^{2}}+\frac{\beta \mu^{2} B_{1}^{2}}{2 G^{4}}+\frac{\gamma \mu^{2} B_{1}^{2}}{2 G^{4}}=0$

Compeering the coefficients of like power of $\alpha$ from both sides of the above equation, we get the following SAE:

$-p \mu A_{1}+2 \lambda \mu^{2} A_{1}+\frac{1}{2} \beta \mu^{2} A_{1}^{2}+\frac{1}{2} \gamma \mu^{2} A_{1}^{2}+p \lambda B_{1}-2 \lambda^{2} \mu B_{1}-2 \beta \lambda \mu A_{1} B_{1}-2 \gamma \lambda \mu A_{1} B_{1}+\frac{1}{2} \beta \lambda^{2} B_{1}^{2}+\frac{1}{2} \gamma \lambda^{2} B_{1}^{2}=0$,

$-p \lambda A_{1}+8 \lambda^{2} \mu A_{1}+\beta \lambda \mu A_{1}^{2}+\gamma \lambda \mu A_{1}^{2}-\beta \lambda^{2} A_{1} B_{1}-\gamma \lambda^{2} A_{1} B_{1}=0$,

$p \mu B_{1}-8 \lambda \mu^{2} B_{1}-\beta \mu^{2} A_{1} B_{1}-\gamma \mu^{2} A_{1} B_{1}+\beta \lambda \mu B_{1}^{2}+\gamma \lambda \mu B_{1}^{2}=0$,

$6 \lambda^{3} A_{1}+\frac{1}{2} \beta \lambda^{2} A_{1}^{2}+\frac{1}{2} \gamma \lambda^{2} A_{1}^{2}=0$,

$-6 \mu^{3} B_{1}+\frac{1}{2} \beta \mu^{2} B_{1}^{2}+\frac{1}{2} \gamma \mu^{2} B_{1}^{2}=0$,

Solving the SAE Eq. (3.11) for $p, A_{0}, A_{1}, B_{1}$ we get several solutions sets as follows:

Set-I.

$p=-4 \lambda \mu, A_{0}=0, A_{1}=-\frac{12 \lambda}{\beta+\gamma}, B_{1}=0$.

Set-II.

$p=-4 \lambda \mu, A_{0}=0, A_{1}=0, B_{1}=\frac{12 \mu}{\beta+\gamma}$

Set-III.

$p=-16 \lambda \mu, A_{0}=0, A_{1}=-\frac{12 \lambda}{\beta+\gamma}, B_{1} \rightarrow \frac{12 \mu}{\beta+\gamma}$

Expending these solution sets, we build the solutions to Eq. (3.7) as follows:

When $\mu \lambda<0$, we obtain the following hyperbolic function solutions: 
$U_{17}(x, y, t)=\frac{12 \sqrt{|\lambda \mu|}}{\beta+\gamma}\left[\frac{b+a \operatorname{Cosh}[2 \sqrt{\lambda \mu} \psi]+a \operatorname{Sinh}[2 \sqrt{\lambda \mu} \psi]}{-b+a \operatorname{Cosh}[2 \sqrt{\lambda \mu} \psi]+a \operatorname{Sinh}[2 \sqrt{\lambda \mu} \psi]}\right] ; \psi=x+y-p \frac{t^{\theta}}{\theta}$

$U_{18}(x, y, t)=-\frac{12 \mu \lambda}{\beta+\gamma \sqrt{|\lambda \mu|}}\left[\frac{-b+a \operatorname{Cosh}[2 \sqrt{\lambda \mu} \psi]+a \operatorname{Sinh}[2 \sqrt{\lambda \mu} \psi]}{b+a \operatorname{Cosh}[2 \sqrt{\lambda \mu} \psi]+a \operatorname{Sinh}[2 \sqrt{\lambda \mu} \psi]}\right] ; \psi=x+y-p \frac{t^{\theta}}{\theta}$

$U_{19}(x, y, t)=\frac{12\left(-\mu+\sqrt{|\lambda \mu|}\left[\frac{b+a \operatorname{Cosh}[2 \sqrt{\lambda \mu} \psi]+a \operatorname{Sinh}[2 \sqrt{\lambda \mu} \psi]}{-b+a \operatorname{Cosh}[2 \sqrt{\lambda \mu} \psi]+a \operatorname{Sinh}[2 \sqrt{\lambda \mu} \psi]}\right]^{2}\right)}{(\beta+\gamma) \frac{\sqrt{|\lambda \mu|}}{\lambda}\left[\frac{b+a \operatorname{Cosh}[2 \sqrt{\lambda \mu} \psi]+a \operatorname{Sinh}[2 \sqrt{\lambda \mu} \psi]}{-b+a \operatorname{Cosh}[2 \sqrt{\lambda \mu} \psi]+a \operatorname{Sinh}[2 \sqrt{\lambda \mu} \psi]}\right]} ; \psi=x+y-p \frac{t^{\theta}}{\theta}$

When $\mu \lambda=0$, we obtain the following rational function solutions:

$U_{20}(x, y, t)=\frac{12 a \lambda}{(\beta+\gamma) \lambda[b+a \psi]} ; \psi=x+y-p \frac{t^{\theta}}{\theta}$

$U_{21}(x, y, t)=\frac{12\left(a^{2} \lambda-\mu \lambda[b+a \psi]^{2}\right)}{a(\beta+\gamma) \lambda[b+a \psi]} ; \psi=x+y-p \frac{t^{\theta}}{\theta}$

When $\mu \lambda>0$, we obtain the following trigonometric function solutions:

$$
\begin{aligned}
& U_{22}(x, y, t)=-\frac{12 \lambda}{\beta+\gamma} \sqrt{\frac{\mu}{\lambda}}\left[\frac{a \operatorname{Cos}[\sqrt{\lambda \mu} \psi]+b \operatorname{Sin}[\sqrt{\lambda \mu} \psi]}{b \operatorname{Cos}[\sqrt{\lambda \mu} \psi]-a \operatorname{Sin}[\sqrt{\lambda \mu} \psi]}\right] ; \psi=x+y-p \frac{t^{\theta}}{\theta} \\
& U_{23}(x, y, t)=\frac{12 \mu}{\beta+\gamma} \sqrt{\frac{\lambda}{\mu}}\left[\frac{b \operatorname{Cos}[\sqrt{\lambda \mu} \psi]-a \operatorname{Sin}[\sqrt{\lambda \mu} \psi]}{a \operatorname{Cos}[\sqrt{\lambda \mu} \psi]+b \operatorname{Sin}[\sqrt{\lambda \mu} \psi]}\right] ; \psi=x+y-p \frac{t^{\theta}}{\theta} \\
& U_{24}(x, y, t)=\frac{12\left(\mu-\lambda \sqrt{\frac{\mu}{\lambda}}\left[\frac{a \operatorname{Cos}[\sqrt{\lambda \mu} \psi]+b \operatorname{Sin}[\sqrt{\lambda \mu} \psi]}{b \operatorname{Cos}[\sqrt{\lambda \mu} \psi]-a \operatorname{Sin}[\sqrt{\lambda \mu}]}\right]^{2}\right)}{(\beta+\gamma) \sqrt{\frac{\mu}{\lambda}}\left[\frac{a \operatorname{Cos}[\sqrt{\lambda \mu} \psi]+b \operatorname{Sin}[\sqrt{\lambda \mu} \psi]}{b \operatorname{Cos}[\sqrt{\lambda \mu} \psi]-a \operatorname{Sin}[\sqrt{\lambda \mu} \psi]}\right]} ; \psi=x+y-p \frac{t^{\theta}}{\theta}
\end{aligned}
$$

\section{Graphical representation}

This section presents the graphical illustration of phi-four and CBS equations. Using the computational software MATLAB, we represent a combinedly $3 \mathrm{D}$ surface and contour plot view of some solutions. All the attained solutions except $U_{20}(x, y, t)$ and $U_{21}(x, y, t)$ represent periodic wave solutions. $U_{20}(x, y, t)$ and $U_{21}(x, y, t)$ are rational function solutions. Each periodic wave is presented for different values of $\theta$. Figure 1-4 represents the periodic waves for phi-four, and CBS equations and Figure 5 represent the rational function solution for the CBS equation. Figures 1-4 are represented for $\theta=0.3,0.6$, and 1 , respectively.

(a) $\theta=0.3$

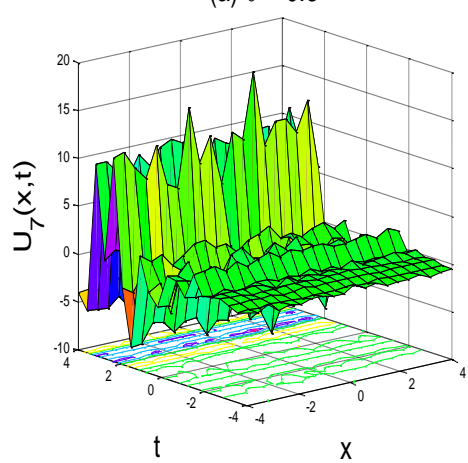

(b) $\theta=0.6$

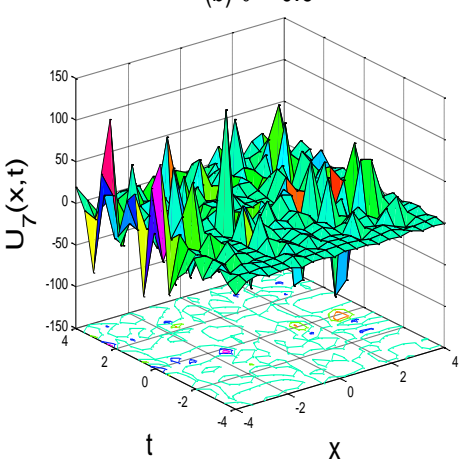

(c) $\theta=1$

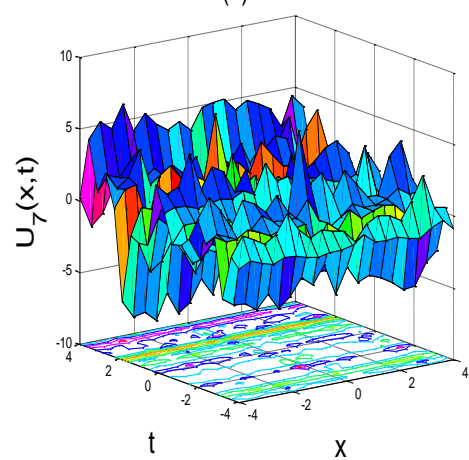

Fig. 1: Periodic Wave of $U_{7}(x, t)$ for the Parameters $a=1, b=1, p=-0.5, \lambda=1, \mu=-1, \beta=0.5, \gamma=0.5$ and $\theta=0.3,0.6$ and 1 Respectively.

(a) $\theta=0.3$

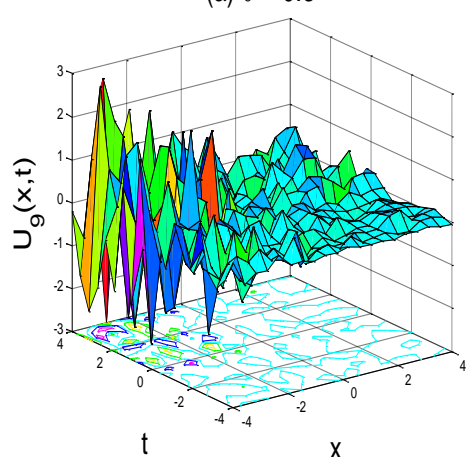

(b) $\theta=0.6$

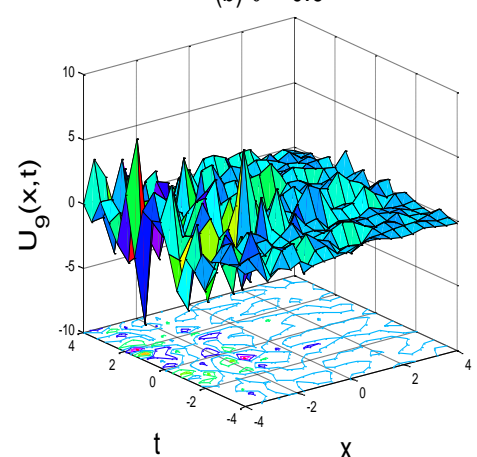

(c) $\theta=1$

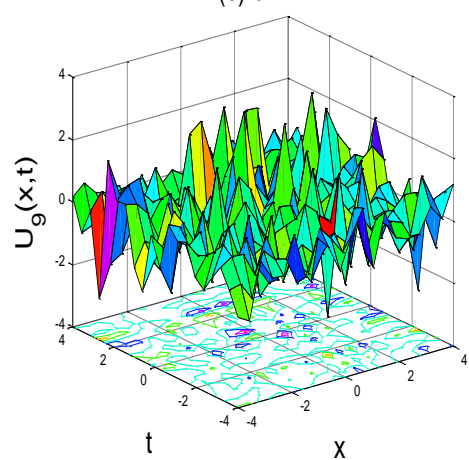

Fig. 2: Periodic Wave of $U_{9}(x, t)$ for the Parameters $a=1, b=1, p=-0.5, \lambda=1, \mu=1, \beta=0.5, \gamma=0.5$ and $\theta=0.3,0.6$ and 1 Respectively. 
(a) $\theta=0.3$

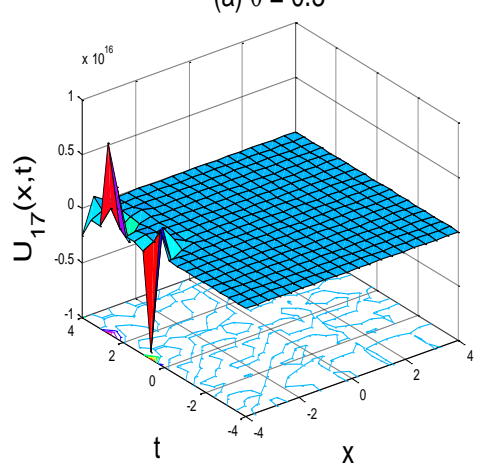

(b) $\theta=0.6$

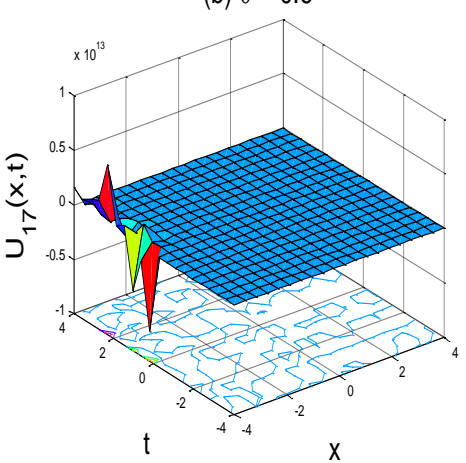

(c) $\theta=1$

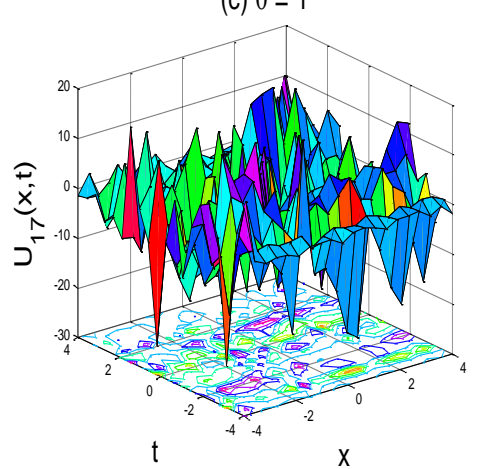

Fig. 3: Periodic Wave of $U_{17}(x, t)$ for the Parameters $a=1, b=1, p=-0.5, \lambda=1, \mu=-1, y=0, \beta=0.5, \gamma=0.5$ and $\theta=0.3,0.6$ and 1 Respectively.

(a) $\theta=0.3$

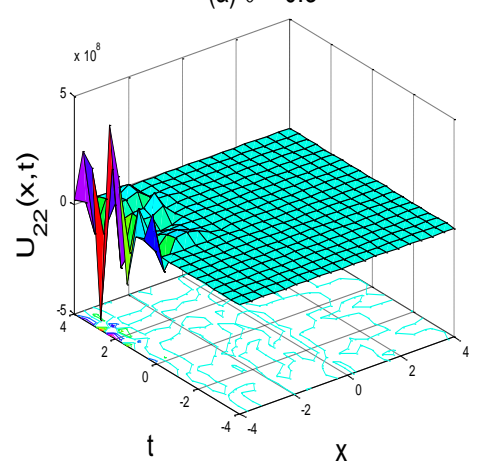

(b) $\theta=0.6$

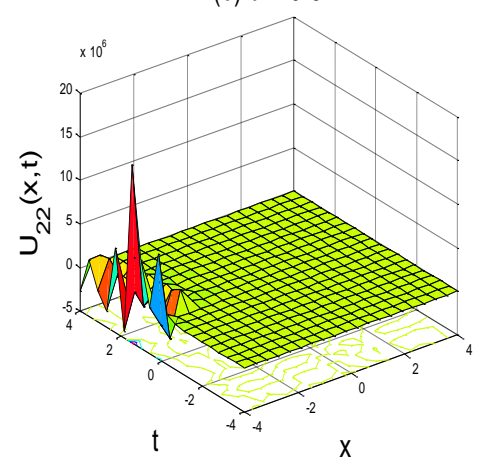

(c) $\theta=1$

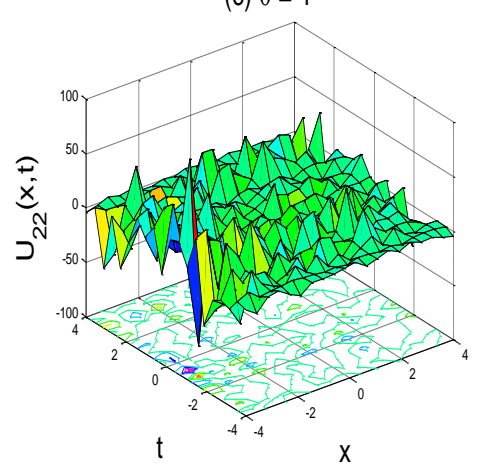

Fig. 4: Periodic Wave of $U_{17}(x, t)$ for the Parameters $a=1, b=1, p=-0.5, \lambda=1, \mu=1, y=0, \beta=0.5, \gamma=0.5$ and $\theta=0.3,0.6$ and 1 Respectively.

(a) $\theta=1$

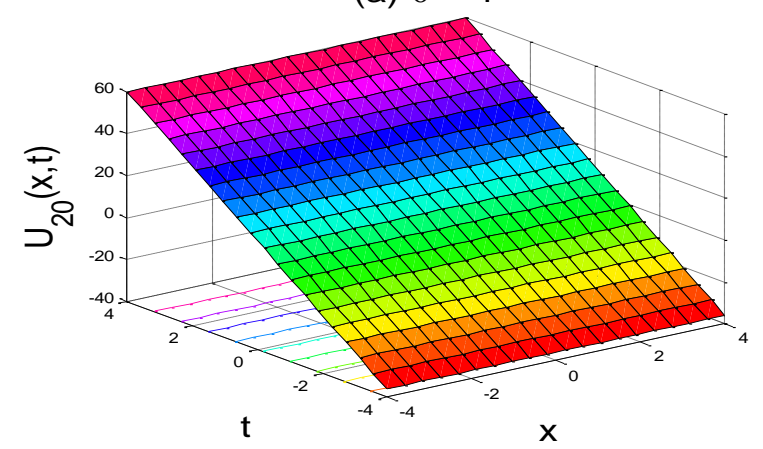

Fig. 5: Rational Function Solution of $U_{20}(x, y, t)$ for the Parameters $a=1, b=1, \lambda=1, \mu=0, y=0, \beta=0.5, \gamma=0.5$ and $\theta=1$.

\section{Conclusion}

In this article, we investigated the periodic wave analysis of phi-four and CBS equations using the $\left(\boldsymbol{G}^{\prime} / \boldsymbol{G}^{2}\right)$-expansion method. Using the mentioned method, we found the exact travelling wave solutions of the phi-four and CBS equations and see that most of the solutions are periodic wave solutions by the hyperbolic function sinh, cosh, and trigonometric function sin and cos. Also, we have some rational function solutions only for the CBS equation. Moreover, we analyzed the periodic wave solutions and the rational function solution for different fractional values of $\boldsymbol{\theta}$, and we found no change of wave characteristics for the change of the fractional value of $\boldsymbol{\theta}$. Eventually, it is noticed that the employed method and the relevant traveling transformation are more realistic, effective, and efficient than the other forms and that they may be used in future productive investigations in mathematical physics and engineering to understand long-wave phenomena.

\section{References}

[1] Miller, M., Kenneth, S., \& Bertram, R. (1993). An introduction to the fractional calculus and fractional differential equations. Wiley.

[2] Alam, L. M. B., Jiang, X., \& Mamun, A. A. (2021). Exact and explicit travelling traveling wave solution to the time-fractional phi-four and (2+1) dimensional CBS equations using the modified extended tanh-function method in mathematical physics. Partial Differential Equations in Applied Mathematics, 4, 100039. https://doi.org/10.1016/j.padiff.2021.100039.

[3] Mamun, A. A., An, T., Shahen, N. H. M., Ananna, S. N., Foyjonnesa, Hossain, M. F., \& Muazu, T. (2020). Exact and explicit travelling-wave solutions to the family of new 3D fractional WBBM equations in mathematical physics. Results in Physics, 19 , 103517. https://doi.org/10.1016/j.rinp.2020.103517.

[4] Mamun, A. A., Ananna, S. N., An, T., Shahen, N. H. M., Asaduzzaman, M., \& Foyjonnesa. (2021). Dynamical behaviour of travelling wave solutions to the conformable time-fractional modified Liouville and mRLW equations in water wave mechanics. Heliyon, 7(8), e07704. https://doi.org/10.1016/j.heliyon.2021.e07704. 
[5] Shahen, N. H. M., Foyjonnesa, Ali, M. S., Mamun, A. A., \& Rahman, M. (2021). Interaction among lump, periodic, and kink solutions with dynamical analysis to the conformable time-fractional Phi-four equation. Partial Differential Equations in Applied Mathematics, 4, 100038. https://doi.org/10.1016/j.padiff.2021.100038.

[6] Shahen, N. H. M., Foyjonnesa, Bashar, M. H., Ali, M. S., \& Mamun, A. A. (2020). Dynamical analysis of long-wave phenomena for the nonlinear conformable space-time fractional (2+1)-dimensional AKNS equation in water wave mechanics. Heliyon, 6(10), e05276. https://doi.org/10.1016/j.heliyon.2020.e05276.

[7] Alam, L. M. B., Xingfang, J., Mamun, A. A., \& Ananna, S. N. (2021). Investigation of lump, soliton, periodic, kink, and rogue waves to the timefractional phi-four and (2+1) dimensional CBS equations in mathematical physics. Partial Differential Equations in Applied Mathematics, 4, 100122. https://doi.org/10.1016/j.padiff.2021.100122.

[8] Mamun, A. A., Ananna, S. N., An, T., Shahen, N. H. M., \& Foyjonnesa. (2021). Periodic and solitary wave solutions to a family of new 3D fractional WBBM equations using the two-variable method. Partial Differential Equations in Applied Mathematics, 3 , 100033. https://doi.org/10.1016/j.padiff.2021.100033.

[9] Mamun, A.-A.-, Ali, M. S., \& Miah, M. M. (2018). A study on an analytic solution 1D heat equation of a parabolic partial differential equation and implement in computer programming. International Journal of Scientific \& Engineering Research, 9(9), 913-921.

[10] Ananna, S. N., \& Mamun, A.-A.-. (2020). Solution of Volterra's integro-differential equations by using variational iteration method. International Journal of Scientific \& Engineering Research, 11(3), 1-9.

[11] Mamun, A.-A.-, Asaduzzaman, M., \& Ananna, S. N. (2019). Solution of eighth order boundary value problem by using variational iteration method. International Journal of Mathematics and Computational Science, 5(2), 13-23.

[12] Mamun, A.-A.-, \& Asaduzzaman, M. (2019). Solution of seventh order boundary value problem by using variational iteration method. International Journal of Mathematics and Computational Science, 5(1), 6-12.

[13] Yaşar, E., Yıldırım, Y., Zhou, Q., Moshokoa, S. P., Ullah, M. Z., Triki, H., Biswas, A., \& Belic, M. (2017). Perturbed dark and singular optical solitons in polarization preserving fibers by modified simple equation method. Superlattices and Microstructures, 111, 487-498. https://doi.org/10.1016/i.spmi.2017.07.004

[14] Khater, M. M. A., \& Kumar, D. (2017). New exact solutions for the time fractional coupled Boussinesq-Burger equation and approximate long water wave equation in shallow water. Journal of Ocean Engineering and Science, 2(3), 223-228. https://doi.org/10.1016/j.joes.2017.07.001.

[15] Ma, W.-X., Huang, T., \& Zhang, Y. (2010). A multiple exp-function method for nonlinear differential equations and its application. Physica Scripta, 82(6), 065003. https://doi.org/10.1088/0031-8949/82/06/065003

[16] Khatun, M. S., Hoque, M. F., \& Rahman, M. A. (2017). Multisoliton solutions, completely elastic collisions and non-elastic fusion phenomena of two PDEs. Pramana, 88(6), 1. https://doi.org/10.1007/s12043-017-1390-3.

[17] Manafian, J., \& Lakestani, M. (2016). The Classification of the Single Traveling Wave Solutions to the Modified Fornberg-Whitham Equation. International Journal of Applied and Computational Mathematics, 3(4), 3241-3252. https://doi.org/10.1007/s40819-016-0288-y.

[18] Lakestani, M., \& Manafian, J. (2017). Application of the ITEM for the modified dispersive water-wave system. Optical and Quantum Electronics, 49(4), 1. https://doi.org/10.1007/s11082-017-0967-X.

[19] Li, Y., Ma, W.-X., \& Zhang, J. E. (2000). Darboux transformations of classical Boussinesq system and its new solutions. Physics Letters A, 275(12), 60-66. https://doi.org/10.1016/S0375-9601(00)00583-1.

[20] Rezazadeh, H., Osman, M. S., Eslami, M., Mirzazadeh, M., Zhou, Q., Badri, S. A., \& Korkmaz, A. (2019). Hyperbolic rational solutions to a variety of conformable fractional Boussinesq-Like equations. Nonlinear Engineering, 8(1), 224-230. https://doi.org/10.1515/nleng-2018-0033.

[21] Lu, D., Seadawy, A., \& Arshad, M. (2017). Applications of extended simple equation method on unstable nonlinear Schrödinger equations. Optik, 140, 136-144. https://doi.org/10.1016/j.ijleo.2017.04.032.

[22] Younis, M., \& Zafar, A. (2013). The modified simple equation method for solving nonlinear Phi-Four equation. International Journal of Innovation and Applied Studies, 2(4), 661-664.

[23] MA, W. E. N.-X. I. U., ZHOU, R. U. G. U. A. N. G., \& GAO, L. I. A. N. G. (2009). EXACT ONE-PERIODIC AND TWO-PERIODIC WAVE SOLUTIONS TO HIROTA BILINEAR EQUATIONS IN (2+1) DIMENSIONS. Modern Physics Letters A, $24(21), 1677-1688$. https://doi.org/10.1142/S0217732309030096.

[24] Eslami, M., \& Rezazadeh, H. (2015). The first integral method for Wu-Zhang system with conformable time-fractional derivative. Calcolo, 53(3), 475-485. https://doi.org/10.1007/s10092-015-0158-8.

[25] Mahmood, S., Mahmood, S., Mahmood, S., \& Mahmood, S. (2014). Mathematical analysis of the Generalized Benjamin and Burger-Kdv Equations via the Extended Trial Equation Method. Journal of the Association of Arab Universities for Basic and Applied Sciences, 16(1), 91-100. https://doi.org/10.1016/j.jaubas.2013.07.005.

[26] Ma, W.-X. (2020). Symbolic Computation of Lump Solutions to a Combined Equation Involving Three Types of Nonlinear Terms. East Asian Journal on Applied Mathematics, 10(4), 732-745. https://doi.org/10.4208/eajam.151019.110420.

[27] Yang, J.-Y., Ma, W.-X., \& Khalique, C. M. (2020). Determining lump solutions for a combined soliton equation in (2+1)-dimensions. The European Physical Journal Plus, 135(6), 1. https://doi.org/10.1140/epjp/s13360-020-00463-Z.

[28] Ma, W.-X., \& Lee, J.-H. (2009). A transformed rational function method and exact solutions to the 3+1 dimensional Jimbo-Miwa equation. Chaos, Solitons \& Fractals, 42(3), 1356-1363. https://doi.org/10.1016/j.chaos.2009.03.043.

[29] Zhou, Q., Mirzazadeh, M., Zerrad, E., Biswas, A., \& Belic, M. (2015). Bright, dark, and singular solitons in optical fibers with spatio-temporal dispersion and spatially dependent coefficients. Journal of Modern Optics, 63(10), 950-954. https://doi.org/10.1080/09500340.2015.1111456.

[30] Bekir, A. (2008). New solitons and periodic wave solutions for some nonlinear physical models by using the sine-cosine method. Physica Scripta, 77(4), 045008. https://doi.org/10.1088/0031-8949/77/04/045008.

[31] Rezazadeh, H. (2018). New solitons solutions of the complex Ginzburg-Landau equation with Kerr law nonlinearity. Optik, 167, $218-227$. https://doi.org/10.1016/j.ijleo.2018.04.026.

[32] Younis, M., \& Zafar, A. (2014). Exact Solution to Nonlinear Differential Equations of Fractional Order via ( $\left.G^{\prime} / G\right)$-Expansion Method. Applied Mathematics, 05(01), 1-6. https://doi.org/10.4236/am.2014.51001.

[33] Mamun, A. A., Shahen, N. H. M., Ananna, S. N., Asaduzzaman, M., \& Foyjonnesa. (2021). Solitary and periodic wave solutions to the family of new 3D fractional WBBM equations in mathematical physics. Heliyon, 7(7), e07483. https://doi.org/10.1016/j.heliyon.2021.e07483.

[34] Rezazadeh, H., Korkmaz, A., Eslami, M., Vahidi, J., \& Asghari, R. (2018). Traveling wave solution of conformable fractional generalized reaction Duffing model by generalized projective Riccati equation method. Optical and Quantum Electronics, 50(3), 150. https://doi.org/10.1007/s11082018-1416-1. 